\title{
The effects of cycle and treadmill desks on work performance and cognitive function in sedentary workers: A review and meta-analysis
}

\author{
Nastja Podrekar $^{\mathrm{a}, \mathrm{b}}$, Žiga Kozinc $^{\mathrm{a}, \mathrm{c}}$ and Nejc Šarabon ${ }^{\mathrm{a}, \mathrm{b}, \mathrm{d}, *}$ \\ ${ }^{a}$ University of Primorska, Faculty of Health Sciences, Izola, Slovenia \\ ${ }^{\mathrm{b}}$ InnoRennew CoE, Izola, Slovenia \\ ${ }^{\mathrm{c}}$ University of Primorska, Andrej Marušič Institute, Koper, Slovenia \\ ${ }^{\mathrm{d}}$ S2P, Science to Practice Ltd., Ljubljana, Slovenia
}

Received 17 September 2018

Accepted 28 April 2019

\begin{abstract}
.
BACKGROUND: Sedentary behavior has been recognized as an important risk factor in the development of several chronic diseases. Active workstations have been proposed as an effective countermeasure. While such interventions likely reduce sedentary time, concerns regarding the effects on work performance and cognitive function remain.

OBJECTIVE: To use meta-analyis to critically evaluate the work performance and cognitive function effects of cycle and treadmill desks as workplace interventions against sedentary behavior.

METHODS: In February 2018, a data search was conducted. Parallel and crossover design studies evaluating workplace interventions compared to conventional seated conditions were included.

RESULTS: Eighteen studies met the inclusion criteria. Both interventions reduced typing speed (cycling: $\mathrm{SMD}=-0.35$, $p=0.04$; treadmill: $\mathrm{SMD}=-0.8, p<0.001)$. The number of typing errors significantly increased during cycling interventions ( $\mathrm{SMD}=0.39, p=0.004)$. No effect was found for the selective attention tests. However, there was an improvement in recall ability (SMD $=0.68, p=0.003$ ).

CONCLUSION: Cycle and treadmill desks affect work performance, but most likely not due to a decrease in cognitive function. Further research is needed to determine whether the degree of work performance decline is acceptable, considering the many positive effects of implementing active workstations in the office environment.
\end{abstract}

Keywords: Active workstation, bike desk, workplace cycling, workplace ergonomics, workplace intervention

*Address for correspondence: Nejc Sarabon, University of Primorska, Faculty of Health Sciences, Polje 42, SI-6310 Izola, Slovenia. Tel.: +38640 429 505; E-mail: nejc.sarabon@fvz.upr.si.

\section{Introduction}

Performing regular physical activity (PA) is not only beneficial for the prevention of several chronic non-communicable diseases [1], such as cardiovascular diseases [2,3] and metabolic syndrome [4, 5], but 
it also promotes cognitive and psychological health [6-8] and could increase job satisfaction and overall quality of life [9]. Individuals achieving high levels of PA express increased self-esteem and report lower stress levels [10]. The effects of PA on cognition are seen at the systemic, molecular and cellular levels [11]. Performing moderate-intensity PA benefits acute concentration-related cognition [12] and could positively affect long-term memory [13]. It has been shown that performing PA increases neurogenesis and accelerates an individual's cognitive development [14]. Moreover, it promotes angiogenesis, synaptogenesis and increases the concentration of brain neurotransmitters [15]. Donnelly \& Lambourne [16] report superior academic success and cognitive function in students performing a larger amount of moderate intensity PA. In addition, reduced sickleave durations and rates have been observed in workers that are physically active [17]. Finally, PA plays an important role in the prevention of cognitive decline and dementia in the elderly [18].

Modern lifestyles promote sedentary behavior to a greater extent than PA (e.g., by requiring computer work), and many employees report lack of time as one of the main reasons for not performing PA $[19,20]$. Therefore, many researchers have begun exploring interventions that incorporate PA into work and other activities. Active workstations, which enable workers to perform PA in the office, are amongst the possible solutions.

Meta-analyses conducted so far have addressed the effects of workplace interventions on sedentary behavior and PA levels [21-26], whereas two metaanalyses have examined the effects on the cognitive function [27, 28]. These meta-analyses included studies with different interventions (active breaks, educational workshops, prompts, sit-to-stand desks, bike and treadmill desks, etc.). However, the specific effects of cycle and treadmill desks on cognitive function and work performance have not been assessed. Moreover, the volume of research regarding workplace cycling and walking has expanded rapidly in recent years, since active workstations indicate positive effects for sedentary workers. Therefore, a re-evaluation of the effects of these interventions is needed.

This review and meta-analysis were conducted to investigate the effects of active workstations permitting continuous PA (i.e., cycle and treadmill desks) on cognitive function and work performance. We hypothesized that both types of intervention will result in improved cognitive function, whereas work performance will not be disturbed to any practical extent.

\section{Methods}

\subsection{Study inclusion criteria}

Studies were included in the meta-analysis if they met the following criteria: i) the study design was a randomized controlled trial, cross-over randomized trial or other (non-randomized) prospective study design including a control group; ii) the study investigated the effects of either cycle or treadmill desks; iii) the participants were sedentary workers (e.g., office workers), aged between 18 and 65, with no medical conditions; iv) the outcome variables included measurements of work performance or cognitive function.

\subsection{Search strategy}

The search was independently conducted by two authors in February 2018 and included PubMed, Web of Science, ScienceDirect, Scopus, BioMedCentral and the Cochrane Central Register of Controlled Trials databases. The search strategy was based on the search terms within four main elements: i) sedentary behavior and its synonyms, ii) workplace physical activity and its synonyms, iii) types of intervention and its synonyms, iv) outcome parameters. Search terms were merged with $\gg \mathrm{AND}$ « and $\gg \mathrm{OR}$ « and terms referring to these elements were used in the title or abstract in all databases. Additionally, reference lists of relevant published systematic review papers were reviewed. The complete list of search terms and the list of systematic reviews are provided in Supplementary file 1 .

\subsection{Assessment of the methodological quality of the studies included}

Two authors independently assessed the methodological quality of the studies included using the ten-level PEDro Scale. The scores were interpreted as high quality (9-10), moderate quality (6-8), sufficient quality (4-5) and low quality ( $\leq 3)$ [29]. The PEDro scale evaluates the study quality based on the following domains: 1.) subjects were randomly allocated to groups; 2.) group allocation was concealed; 3.) important baseline prognostic indicators were similar among groups; 4.) all subjects were blinded; 5.) 
all participating therapists were blinded; 6.) all assessors that measured at least one key outcome were blinded; 7.) measures of at least one key outcome were obtained from more than $85 \%$ of the subjects initially allocated to the groups; 8 .) all subjects whose outcome measures were available received the treatment or the control condition as allocated or, where this was not the case, the data for at least one key outcome was analyzed by "intention to treat"; 9.) the results of between-group statistical comparisons are reported for at least one key outcome; 10.) the study provides both point measures and measures of variability for at least one key outcome [30]. The PEDro scale was used to determine study eligibility in this meta-analysis, as it covers the four main types of biases indicated by the Cochrane Collaboration (the agency providing recommendations for health care interventions): i) selection bias; ii) performance bias; iii) attrition bias; iiii) detection bias [31]. Potential disagreements between the assessors on the final outcome were resolved by the third author.

\subsection{Data extraction}

The data extracted from each study included: the first author's last name, the year of publication, the study design, the type of intervention, the duration and the intensity of the intervention, the location of the study, the sample size, the participants' age and anthropometric data, as well as the means and standard deviations for all relevant outcome measures. The extraction was performed by all the authors separately to avoid errors. The data was compiled in Microsoft Excel 2016 (Microsoft Corp., Redmond, WA) for further analysis. When the data reported in the full-text papers was insufficient, the authors were contacted and asked to provide the missing data.

\subsection{Data analysis}

All data analyses were conducted using Review Manager, version 5.3 (Copenhagen: The Nordic Cochrane Centre, The Cochrane Collaboration). Studies that reported results for the same measurements were pooled into the meta-analysis using a random effects model with the inverse variance method. When possible, the mean difference (MD) method was used. However, due to the heterogeneity of the cognitive function as well as the work performance assessment tools, the standardized mean difference (SMD) was also used to express the difference between the intervention and the control groups.
Individual studies were sorted for subgroup analysis based on intervention type (cycle or treamdill desks). Statistical heterogeneity between the studies was assessed using the $\mathrm{I}^{2}$ test; $<30 \%=$ negligible; $30 \%-75 \%=$ moderate; $\quad>75 \%=$ considerable [32]. The threshold level for statistical significance was set at $p<0.05$.

\section{Results}

\subsection{Literature search}

The database search identified 1034 potentially relevant studies and seven more were collected from the reference lists of existing systematic reviews. After removal of duplicates, the abstracts of 875 articles were screened reducing the dataset to 153 studies for full-text analysis. Finally, 18 studies were included in the meta-analyses. A flowchart of the database search is depicted in Fig. 1.

Among the 18 studies included, two were randomized controlled trials [33, 34], two were nonrandomized parallel group design studies $[35,36]$ and 14 were crossover studies [37, 38, 47-50, 39-46]. The average age of participants ( 225 males, 376 females, 121 unknown) was $31.0 \pm 6.4$ years and their average BMI was $24.4 \pm 3.6$. The study quality was moderate (average outcome 5.2 / 10, median outcome 5), as seen in Supplementary File 3.

\subsection{The effects of the interventions on work performance}

Altogether, nine studies examining work performance were included in the meta-analyses. Five studies evaluated cycle desks [33, 40, 41, 47, 50], two studies evaluated treadmill desks $[34,44]$ and two studies evaluated both types of interventions [42, 49]. Two meta-analyses were conducted evaluating typing speed and the number of typing errors in the intervention group compared to the control group in the sitting condition.

The first typing speed meta-analysis included eight studies (363 participants). All studies were performed in a laboratory environment. The Forest Plot (Fig. 2) shows the pooled effect size separately for the cycle and treadmill desk interventions and the total effect size for both interventions together. Studies which included both cycle and treadmill desk interventions $[42,49]$ were included in both Subgroups based on the intervention type. Four studies performed the 


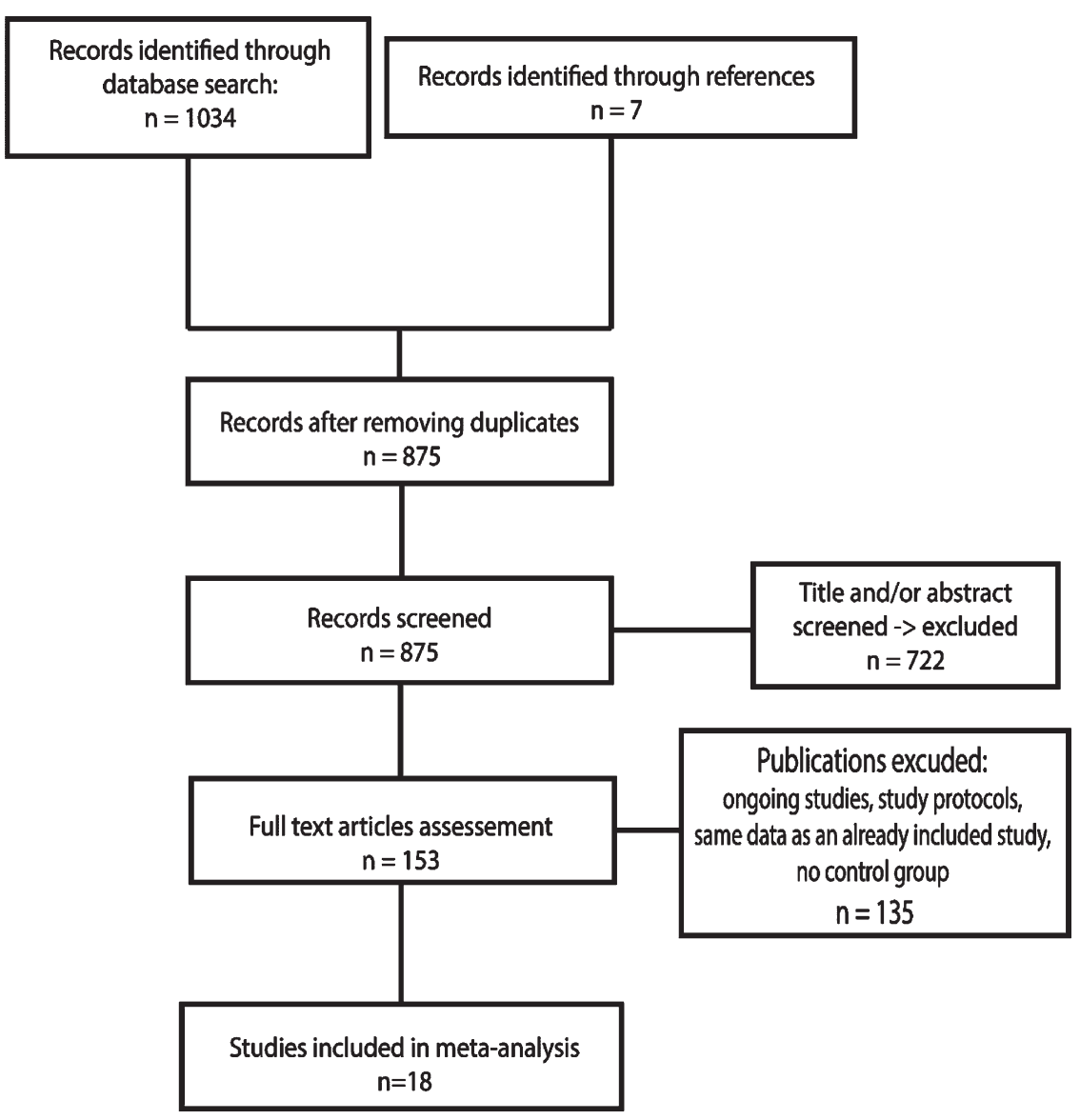

Fig. 1. Flowchart of database search.

intervention condition at more than one intensity [41, 42, 45, 49]. In these cases, the results for each intensity level were included in the meta-analysis separately. Descriptions of the intensity level of each intervention are provided in Fig. 2.

The pooled effect size indicated a statistically significant negative effect on typing speed $\left(\mathrm{SMD}=-0.60,[-0.85,-0.35], p<0.001, \mathrm{I}^{2}=62 \%\right)$. Analysis of the intervention subgroups revealed that both cycling (SMD $=-0.35,[-0.68,-0.02], p=0.04$, $\left.\mathrm{I}^{2}=50 \%\right)$ and treadmill interventions $(\mathrm{SMD}=-0.80$, $\left.[-1.12,-0.51], p<0.001, \mathrm{I}^{2}=55 \%\right)$ caused a reduction in typing speed. The differences between the subgroups were statistically significant $(p=0.04)$. Statistical heterogeneity between included studies was moderate $\left(\mathrm{I}^{2}=62 \%, p<0.001\right)$.

Four studies with a total of 167 participants were included in the assessment of the effect of cycling and treadmill interventions on the number of typing errors (see Supplementary File 4). Each of these studies were performed in a laboratory environment.
Two of the studies $[33,42]$ included both cycle and treadmill desk interventions and two of the studies $[42,47]$ investigated the effects at different intensity levels. The pooled effect size showed a significant effect on the number of errors, which was greater in the intervention group $(\mathrm{SMD}=0.37,[0.15,0.58]$, $\left.p=0.0009, \mathrm{I}^{2}=0 \%\right)$. Subgroup analysis showed that the negative effect is only present in cycling interventions $\left(\mathrm{SMD}=0.39,[0.13,0.66], p=0.004, \mathrm{I}^{2}=0\right.$ $\%)$, but not in treadmill interventions $(p=0.16)$, although there were no statistically significant differences between the subgroups $(p=0.90)$. Statistical heterogeneity was not present $\left(\mathrm{I}^{2}=0 \%, p=0.62\right)$.

\subsection{The effects of interventions on cognitive function}

Nine studies were included in the cognitive function meta-analyses [35-39, 44, 46, 48, 50]. The studies evaluated cognitive function with different tests. The meta-analyses were conducted for the Eriksen 


\begin{tabular}{|c|c|c|c|c|}
\hline \multirow{2}{*}{$\begin{array}{l}\text { Study or Subgroup } \\
\text { Bike Desk }\end{array}$} & \multicolumn{2}{|c|}{ Std. Mean Difference } & \multicolumn{2}{|c|}{$\begin{array}{l}\text { Std. Mean Difference } \\
\text { IV, Random, } 95 \% \mathrm{Cl}\end{array}$} \\
\hline & & & & \\
\hline Carr 2014 & $6.3 \%$ & $-0.04[-0.67,0.58]$ & & \\
\hline Cho 2014 a & $4.8 \%$ & $-0.85[-1.70,-0.01]$ & $\longrightarrow$ & \\
\hline Cho 2014 b & $3.9 \%$ & $-1.92[-2.92,-0.93]$ & $\longrightarrow$ & \\
\hline Commissaris 2014 a.1 & $5.6 \%$ & $-0.12[-0.84,0.60]$ & - & \\
\hline Commissaris 2014 a.2 & $5.6 \%$ & $-0.07[-0.78,0.65]$ & $\longrightarrow$ & - \\
\hline Straker 2009 a.1 & $7.3 \%$ & $-0.29[-0.79,0.22]$ & 7 & \\
\hline Straker 2009 a.2 & $7.3 \%$ & $-0.25[-0.76,0.26]$ & & \\
\hline $\begin{array}{l}\text { Torbeyns } 2016 \mathrm{~b} \\
\text { Subtotal }(95 \% \mathrm{Cl})\end{array}$ & $\begin{array}{r}6.7 \% \\
47.4 \%\end{array}$ & $\begin{array}{r}-0.05[-0.63,0.53] \\
-0.35[-0.68,-0.02]\end{array}$ & $\overrightarrow{ }$ & \\
\hline \multicolumn{5}{|c|}{$\begin{array}{l}\text { Heterogeneity: } \operatorname{Tau}^{2}=0.11 ; \mathrm{Ch}^{2}=13.89, \mathrm{df}=7(P=0.05) ; I^{2}=50 \% \\
\text { Test for overall effect: } Z=2.09(P=0.04)\end{array}$} \\
\hline \multicolumn{5}{|l|}{ Treadmill Desk } \\
\hline Commissaris 2014 & $5.6 \%$ & $-0.34[-1.06,0.38]$ & $\rightarrow$ & \\
\hline Funk 2014 a & $6.0 \%$ & $-1.64[-2.30,-0.98]$ & 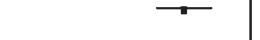 & \\
\hline Funk 2014 b & $6.7 \%$ & $-0.64[-1.23,-0.06]$ & 7 & \\
\hline Funk 2014 c & $6.1 \%$ & $-1.55[-2.20,-0.90]$ & 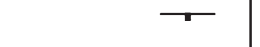 & \\
\hline John 2009 & $6.3 \%$ & $-0.34[-0.96,0.28]$ & 7 & \\
\hline Larson 2015 & $7.6 \%$ & $-0.71[-1.17,-0.24]$ & - & \\
\hline Straker 2009 b.1 & $7.1 \%$ & $-0.68[-1.20,-0.16]$ & $\rightarrow$ & \\
\hline $\begin{array}{l}\text { Straker } 2009 \mathrm{~b} .2 \\
\text { Subtotal }(95 \% \mathrm{Cl})\end{array}$ & $\begin{array}{r}7.1 \% \\
\mathbf{5 2 . 6 \%}\end{array}$ & $\begin{array}{l}-0.71[-1.24,-0.19] \\
-0.82[-1.12,-0.51]\end{array}$ & $\vec{\nabla}$ & \\
\hline \multicolumn{5}{|c|}{$\begin{array}{l}\text { Heterogeneity: } \text { Tau }^{2}=0.11 ; \mathrm{Chi}^{2}=15.61, \mathrm{df}=7(P=0.03) ;\left.\right|^{2}=55 \% \\
\text { Test for overall effect: } Z=5.18(P<0.00001)\end{array}$} \\
\hline Total $(95 \% \mathrm{Cl})$ & $100.0 \%$ & $-0.60[-0.85,-0.35]$ & 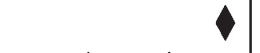 & \\
\hline $\begin{array}{l}\text { Heterogeneity: Tau }{ }^{2}=0 . \\
\text { Test for overall effect: } Z \\
\text { Test for subaroup differe }\end{array}$ & $\begin{array}{l}16 ; \mathrm{Chi}^{2}=3 \\
=4.69(P< \\
\text { nces: } \mathrm{Chi}^{2}=\end{array}$ & $\begin{array}{l}8.89, d f=15(P=0.0005) ; I^{2}=62 \% \\
.00001) \\
4.13, d f=1(P=0.04), I^{2}=75.8 \%\end{array}$ & $\begin{array}{ccc}-4 & -2 & 0 \\
\text { Favours [experimental] }\end{array}$ & $\begin{array}{ccc}1 & 1 & 1 \\
\text { Favours [control] }\end{array}$ \\
\hline
\end{tabular}

Fig. 2. Effects of cycle and treadmill desks on the Typing speed.

Flanker test, the Stroop test and the Recall ability test. All tests were made in comparison to a control condition of sitting.

Two studies (75 participants) examining recall abilities were included in the analysis (Fig. 3). Both studies investigated treadmill interventions and were performed in a laboratory environment. There was a significant positive pooled effect favouring the intervention $\left(\mathrm{SMD}=0.68,[0.18,0.87], p=0.003, \mathrm{I}^{2}=40\right.$ $\%)$, whereas heterogeneity of the studies was negligible $\left(\mathrm{I}^{2}=0 \%, p=0.16\right)$.

Four independent meta-analyses were run to examine the effects of the interventions on the Eriksen Flanker test (see Supplementary File 4), including assessment of completition time and the accuracy of the two test versions (with congruent and incongruent stimulus). None of the four analyses showed any statistically significant effects ( $p=0.27-0.93)$.

Two independent meta-analyses were conducted to assess the effects of the intervention on the Stroop test (see Supplementary file 4). Stroop test accuracy was included in three studies. There was no effect on the accuracy of the test for the cycling interventions $(p=0.50)$ or the treadmill interventions $(p=0.20)$. As with the accuracy of the test, there was no effect on completition time for the cycling interventions $(p=0.93)$ or the treadmill interventions $(p=0.30)$. 


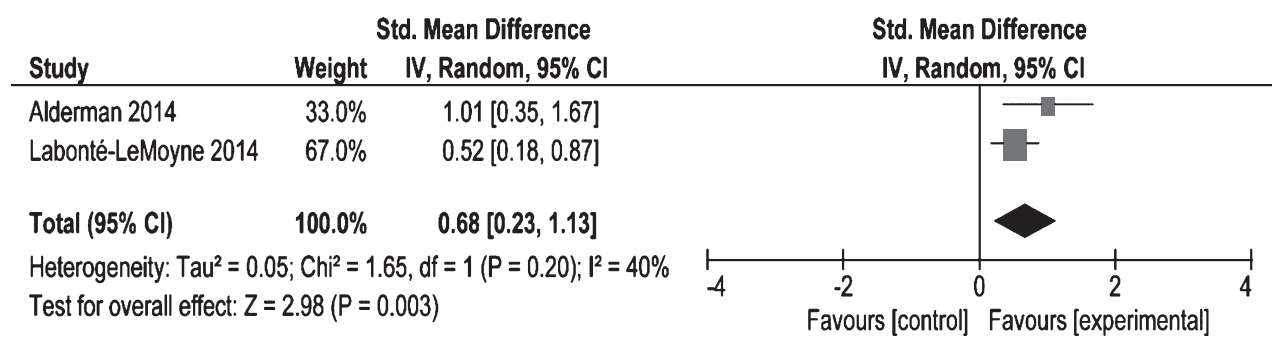

Fig. 3. Effects of treadmill desk interventions on the Recall abilities.

\section{Discussion}

The aim of this paper was to meta-analytically evaluate the work performance and cognitive function effects of interventions that allow typically sedentary workers to perform activities continuously (e.g., walking or cycling) during worktime. In these metaanalyses active wokstations significantly reduced typing speed and increased the number of typing errors, however, there was an improvement in recall ability. No effect was found for the selective attention tests.

Studies included in the meta-analyses substantially differed regarding the intensity levels of cycling and walking. Torbeyns et al. [35] did not define the cycling intensity in advance whereas in the study by Straker et al. [49], the intensity of cycling was set at 5 Watts for the low intensity test and 30 Watts for the high intensity cycling test. In this study there was a significant decrease in typing speed when cycling at low intensity and no change in typing speed when cycling at high intensity compared to the seated condition. In contrast, Cho et al. [41] showed no significant decrease in typing speed at low intensity cycling (10 Watts) and a significant decrease at high intensity cycling ( 25 Watts). Heart rate reserve was used to define intensity in two studies $[42,50]$, which included intensity ranges from $25 \%$ to $40 \%$ of heart rate reserve. Since all the included studies were performed in a laboratory environment, it would be of interest to evaluate work performance in a real environment at different intensities and at the user's preferred cycling intensity.

Similar inconsistencies were found for walking intensities. Interestingly, Funk et al. [45] report a decline in typing speed while walking at 1.3 and $3.2 \mathrm{~km} / \mathrm{h}$ on a treadmill, but not at $2.25 \mathrm{~km} / \mathrm{h}$. Similarly, Straker et al. [49] demonstrate a decrease in typing performance when walking at $1.6 \mathrm{~km} / \mathrm{h}$ (50 words/minutes) and $3.2 \mathrm{~km} / \mathrm{h}(49.6$ words/minutes) compared to the sitting condition (54.4 words/minutes). The results of the individual studies show varied effects of active workstations on work performance. However, the meta-analyses conducted in this paper indicate a negative effect of cycle and treadmill desks on work performance in sedentary workers. Typing speeds decreased when using cycle and treadmill desks. The latter effect was more pronounced with treadmill desks than for cycling stations $(p=0.03)$. Since a seated cycling posture results in smaller upper body movement compared to walking, this result is understandable. The number of typing errors significantly increased while cycling but not while using the treadmill desk. However, only two studies were included in the treamdill desk analysis and four in the cycle desk analyses. These results are in accordance with the previous meta-analysis by Cao et al. [28], which revealed that active workstations decrease typing speed and impair computer-mouse work accuracy. Cao et al. [28] also report that workplace PA noticeably affect motor skills but do not alter cognitive function. Nevertheless, the results are not completely comparable since Cao et al. [28] did not conduct a separate meta-analysis for cycling and walking conditions but combined different types of interventions. Our results are in concordance with a systematic review by Ojo et al. [28] who identified trivial, small and large decrease in work performance when using cycle desks (depending on cycling intensity).

Reduced work performance could arise from the complexity of simultaneously performing gross and fine movements. Studies investigating how much attention capacity walking requires are inconclusive [51, 52]. Since our results did not show a decrease in cognitive function, the reason for poorer work performance likely lies elsewhere. It must be considered that in most of the studies participants lacked familiarity with the active workstations. It has been shown that performing two independent motor 
tasks simultaneously may be improved with practice [53-55]. This implies that work performance could be restored in long-term use of active workstations.

Our meta-analyses showed positive or neutral cognitive function effects on sedentary workers when using active workstations. Meta-analyses were conducted for the Recall test (measuring recall abilities), Stroop, and the Eriksen Flanker tests, which evaluate processing speed, executive abilities and selective attention [37]. Treadmill interventions improved recall abilities. This supports the findings of van Praag (2008) who reported that combining PA and cognitive work enhances memory and increases learning abilities. However, the two studies included indicate only acute effects of walking and the long-term effects of treadmill desks on recall abilities remain unknown. Additionally, both studies were performed in a laboratory environment which can differ considerably from an office environment. Our results showed no intervention effects on either the Eriksen Flanker or the Stroop tests. Therefore, performing PA during work may not impair or improve selective attention and concentration. These results confirm the findings of Torbeyns, Bailey, Bos, \& Meeusen [56], who also report no change in either of the these tests. Similarly, Cao et al. [28] found no changes in selective attention while performing workplace PA. Loprinzi \& Kane [12] indicate an increase in concentrationrelated cognition when performing 30 minutes of moderate intensity PA and similar findindgs were drawn up and explained in a review by Ratey \& Loehr [11], who explain that on systematic levels, performing PA improves attention, learning and memory. Regarding the positive effects of PA on cognition, it would be of interest to evaluate the effects of cycling or walking on innovative thinking. If so, despite the negative effect on work performance, work and cognitive productivity may still be enhanced.

There have been several reviews and meta-analyses published in recent years evaluating the effects of active workstations on different parameters in workers. Most of the publications focused on reducing sedentary behavior and increasing PA in workers and included studies with different types of interventions. For example, Chu et al. [21] included mixed, environmental and educational interventions, whereas Martin et al. [22] included also life style interventions like diets etc. Reed et al. [25] focused on studies that included mostly female participants and evaluated the effects on the cardio-metabolic parameters. In a review by Shrestha et al. [26], the focus was on sedentary behavior and energy expenditure. Two reviews [27, 28] focused on the effects of active workstations on work and cognitive performance but did not evaluate the speific effects of cycle and treadmill desks. Hence, no review and meta-analyses to date evaluated the effects of cycle and treadmill desks alone on work performance and cognitive function.

In conclusion, workstations that allow users to perform cycling and walking activities decrease work performance, but not cognitive function. Future studies, using longer intervention periods, performed in a real environment are desired to elucidate the longterm effects of cycle and treadmill desks during work.

\subsection{Limitations}

Only peer-reviewed articles were included in this paper. Studies such as theses, unpublished papers and conference papers were not included. This ensured that the included studies were of acceptable quality, although it could have induced selection bias. Other limitations are lack of real environment studies and the heterogeneity among studies regarding the number of participants, the intensity of the interventions and the experimental design. Therefore, the pooled effects sizes should be interpreted with caution, despite using random effects model and presenting the results as standardized mean differences. Only a limited number of variables were tracked in mutiple studies (e.g. typing speed), while certain variables were only included in a limited number of studies (e.g. recall abilities). For such variables, more studies are needed to provide solid conclusions.

\section{Acknowledgments}

The authors gratefully acknowledge the European Commission for funding the InnoRenew $\mathrm{CoE}$ project (Grant Agreement \#739574) under the Horizon2020 Widespread-Teaming program.

\section{Conflict of interest}

The authors state no conflict of interest.

\section{References}

[1] Fernandez-Navarro P, Aragones MT, Ley V. Leisure-time physical activity and prevalence of non-communicable pathologies and prescription medication in Spain. PLoS One. 2018;13(1):e0191542. 
[2] Wahid A, Manek N, Nichols M, Kelly P, Foster C, Webster P, Kaur A, Friedemann Smith C, Wilkins E, Rayner M, Roberts N, Scarborough P. Quantifying the association between physical activity and cardiovascular disease and diabetes: A systematic review and meta-analysis. J Am Heart Assoc. 2016;5(9):e002495.

[3] Adams RA, Higgins T, Potter S, Evans S-A. The effect of physical activity on haematological predictors of cardiovascular risk: Evidence of a dose response. Clin Hemorheol Microcirc. 2012;52(1):57-65.

[4] Lin C-H, Chiang S-L, Yates P, Lee M-S, Hung Y-J, Tzeng W-C, Chiang LC. Moderate physical activity level as a protective factor against metabolic syndrome in middle-aged and older women. J Clin Nurs. 2015;24(9-10):1234-45.

[5] Zhang D, Liu X, Liu Y, Sun X, Wang B, Ren Y, Zhao Y, Zhou J, Han C, Yin L, Zhao J, Shi Y, Zhang M, Hu D. Leisure-time physical activity and incident metabolic syndrome: A systematic review and dose-response meta-analysis of cohort studies. Metabolism. 2017;75:36-44.

[6] Poirel E. Psychological benefits of physical activity for optimal mental health. Sante Ment Que. 42(1):147-64.

[7] Phillips C. Lifestyle Modulators of Neuroplasticity: How Physical Activity, Mental Engagement, and Diet Promote Cognitive Health during Aging. Neural Plast. 2017;2017:122.

[8] Yamazaki F, Yamada H, Morikawa S. Influence of an 8-week exercise intervention on body composition, physical fitness, and mental health in female nursing students. J UOEH. 2013;35(1):51-8.

[9] Arslan SS, Alemdaroğlu İ, Ayşe Karaduman A, Yilmaz ÖT. The effects of physical activity on sleep quality, job satisfaction, and quality of life in office workers. Work. 2019; 1-5.

[10] Paolucci EM, Loukov D, Bowdish DME, Heisz JJ. Exercise reduces depression and inflammation but intensity matters. Biol Psychol. 2018;133:79-84.

[11] Ratey JJ, Loehr JE. The positive impact of physical activity on cognition during adulthood: A review of underlying mechanisms, evidence and recommendations. Rev Neurosci. 2011;22(2):171-85.

[12] Loprinzi PD, Kane CJ. Exercise and Cognitive Function. Mayo Clin Proc. 2015;90(4):450-60.

[13] Etnier JL, Wideman L, Labban JD, Piepmeier AT, Pendleton DM, Dvorak KK, Becofsky K. The Effects of Acute Exercise on Memory and Brain-Derived Neurotrophic Factor (BDNF). J Sport Exerc Psychol. 2016;38(4):331-40.

[14] van Praag H. Neurogenesis and exercise: Past and future directions. Neuromolecular Med. 2008;10(2):128-40.

[15] Lista I, Sorrentino G. Biological mechanisms of physical activity in preventing cognitive decline. Cell Mol Neurobiol. 2010;30(4):493-503.

[16] Donnelly JE, Lambourne K. Classroom-based physical activity, cognition, and academic achievement. Prev Med (Baltim). 2011;52:S36-42.

[17] van den Heuvel SG, Boshuizen HC, Hildebrandt VH, Blatter BM, Ariëns GA, Bongers PM. Effect of sporting activity on absenteeism in a working population. Br J Sports Med. 2005;39(3):e15.

[18] Laurin D, Verreault R, Lindsay J, MacPherson K, Rockwood $\mathrm{K}$. Physical activity and risk of cognitive impairment and dementia in elderly persons. Arch Neurol. 2001;58(3):498504.

[19] Mailey EL, Huberty J, Dinkel D, McAuley E. Physical activity barriers and facilitators among working mothers and fathers. BMC Public Health. 2014;14(1):657.
[20] Parry SM, Knight LD, Connolly B, Baldwin C, Puthucheary Z, Morris P, Mortimore J, Hart N, Denehy L, Granger CL. Factors influencing physical activity and rehabilitation in survivors of critical illness: A systematic review of quantitative and qualitative studies. Intensive Care Med. 2017;43(4):531-42.

[21] Chu AHY, Ng SHX, Tan CS, Win AM, Koh D, MüllerRiemenschneider F. A systematic review and meta-analysis of workplace intervention strategies to reduce sedentary time in white-collar workers. Obes Rev. 2016;17(5):467-81.

[22] Martin A, Fitzsimons C, Jepson R, Saunders DH, van der Ploeg HP, Teixeira PJ, Gray CM, Mutrie N. Interventions with potential to reduce sedentary time in adults: Systematic review and meta-analysis. Br J Sports Med. 2015;49(16):1056-63.

[23] Neuhaus M, Eakin EG, Straker L, Owen N, Dunstan DW, Reid N, Healy GN. Reducing occupational sedentary time: A systematic review and meta-analysis of evidence on activity-permissive workstations. Obes Rev An Off J Int Assoc Study Obes. 2014;15(10):822-38.

[24] Prince SA, Saunders TJ, Gresty K, Reid RD. A comparison of the effectiveness of physical activity and sedentary behaviour interventions in reducing sedentary time in adults: A systematic review and meta-analysis of controlled trials. Obes Rev. 2014;15(11):905-19.

[25] Reed JL, Prince SA, Elliott CG, Mullen K-A, Tulloch HE, Hiremath S, Cotie LM, Pipe AL, Reid RD. Impact of workplace physical activity interventions on physical activity and cardiometabolic health among working-age women. Circ Cardiovasc Qual Outcomes. 2017;10(2):e003516.

[26] Shrestha N, Ijaz S, Kukkonen-Harjula KT, Kumar S, Nwankwo CP. Workplace interventions for reducing sitting at work. Cochrane Database of Systematic Reviews. 2015;1:CD010912.

[27] Ojo SO, Bailey DP, Chater AM, Hewson DJ. The impact of active workstations on workplace productivity and performance: A systematic review. Int J Environ Res Public Health. 2018;15(3):E417.

[28] Cao C, Liu Y, Zhu W, Ma J. Effect of active workstation on energy expenditure and job performance: A systematic review and meta-analysis. J Phys Act Health. 2016;13(5):562-71.

[29] Hariohm K, Prakash V, Saravankumar J. Quantity and quality of randomized controlled trials published by Indian physiotherapists. Perspect Clin Res. 2015;6(2):91-7.

[30] Maher CG, Sherrington C, Herbert RD, Moseley AM, Elkins M. Reliability of the PEDro scale for rating quality of randomized controlled trials. Phys Ther. 2003;83(8):71321.

[31] Furlan AD, Pennick V, Bombardier C, van Tulder M, Editorial Board, Cochrane Back Review Group. 2009 Updated Method Guidelines for Systematic Reviews in the Cochrane Back Review Group. Spine. 2009;34(18):1929-41.

[32] Higgins JPT, Thompson SG. Quantifying heterogeneity in a meta-analysis. Stat Med. 2002;21(11):1539-58.

[33] Sliter M, Yuan Z. Workout at work: Laboratory test of psychological and performance outcomes of active workstations. J Occup Health Psychol. 2015;20(2):259-71.

[34] Larson MJ, LeCheminant JD, Hill K, Carbine K, Masterson T, Christenson E. Cognitive and Typing Outcomes Measured Simultaneously with Slow Treadmill Walking or Sitting: Implications for Treadmill Desks. Thompson D, editor. PLoS One. 2015;10(4):e0121309.

[35] Torbeyns T, de Geus B, Bailey S, De Pauw K, Decroix L, Van Cutsem J, Meeusen R. Bike desks in the office: Physi- 
cal health, cognitive function, work engagement, and work performance. J Occup Environ Med. 2016;58(12):1257-63.

[36] Labonté-LeMoyne É, Santhanam R, Léger P-M, Courtemanche F, Fredette M, Sénécal S. The delayed effect of treadmill desk usage on recall and attention. Comput Human Behav. 2015;46:1-5.

[37] Alderman BL, Olson RL, Mattina DM. Cognitive function during low-intensity walking: A test of the treadmill workstation. J Phys Act Heal. 2014;11(4):752-8.

[38] Bergouignan A, Legget KT, De Jong N, Kealey E, Nikolovski J, Groppel JL, Jordan C, O’Day R, Hill JO, Bessesen DH. Effect of frequent interruptions of prolonged sitting on self-perceived levels of energy, mood, food cravings and cognitive function. Int $\mathrm{J}$ Behav Nutr Phys Act. 2016;13(1):113.

[39] Botter J, Ellegast RP, Burford E-M, Weber B, Könemann $\mathrm{R}$, Commissaris DACM. Comparison of the postural and physiological effects of two dynamic workstations to conventional sitting and standing workstations. Ergonomics. 2016;59(3):449-63.

[40] Carr L, Maeda H, Luther B, Rider P, Tucker S, Leonhard C. Acceptability and effects of a seated active workstation during sedentary work: A proof of concept study. Int J Work Heal Manag. 2014;7:2-15.

[41] Cho J, Freivalds A, Rovniak L, Sung K, Hatzell J. Using a desk-compatible recumbent bike in an office workstation. Proc hum factors ergon soc annu meet. 2014;58(1):1662-6.

[42] Commissaris DACM, Könemann R, Hiemstra-van Mastrigt S, Burford E-M, Botter J, Douwes M, Ellegast RP. Effects of a standing and three dynamic workstations on computer task performance and cognitive function tests. Appl Ergon. 2014;45(6):1570-8

[43] Ehmann PJ, Brush CJ, Olson RL, Bhatt SN, Banu AH, Alderman BL. Active Workstations Do Not Impair Executive Function in Young and Middle-Age Adults. Med Sci Sports Exerc. 2017;49(5):965-74.

[44] Elmer SJ, Martin JC. A cycling workstation to facilitate physical activity in office settings. Appl Ergon. 2014;45(4):1240-6.

[45] Funk RE, Taylor ML, Creekmur CC, Ohlinger CM, Cox RH, Berg WP. Effect of walking speed on typing performance using an active workstation. Percept Mot Skills. 2012;115(1):309-18.
[46] John D, Bassett D, Thompson D, Fairbrother J, Baldwin D. Effect of using a treadmill workstation on performance of simulated office work tasks. J Phys Act Health. 2009;6(5):617-24.

[47] Koren K, Pišot R, Šimunič B. Active workstation allows office workers to work efficiently while sitting and exercising moderately. Appl Ergon. 2016;54:83-9.

[48] Ohlinger CM, Horn TS, Berg WP, Cox RH. The effect of active workstation use on measures of cognition, attention, and motor skill. J Phys Act Health. 2011;8(1):119-25.

[49] Straker L, Levine J, Campbell A. The effects of walking and cycling computer workstations on keyboard and mouse performance. Hum Factors. 2009;51(6):831-44.

[50] Torbeyns T, de Geus B, Bailey S, De Pauw K, Decroix L, Van Cutsem J, Meeusen R. Cycling on a Bike Desk Positively Influences Cognitive Performance. Allen P, editor. PLoS One. 2016;11(11):e0165510.

[51] Hollman JH, Kovash FM, Kubik JJ, Linbo RA. Age-related differences in spatiotemporal markers of gait stability during dual task walking. Gait Posture. 2007;26(1):113-9.

[52] Yogev-Seligmann G, Hausdorff JM, Giladi N. The role of executive function and attention in gait. Mov Disord Off $\mathrm{J}$ Mov Disord Soc. 2008;23(3):329-42; 472.

[53] Pashler H, Johnston JC, Ruthruff E. Attention and performance. Annu Rev Psychol. 2001;52:629-51.

[54] Schumacher EH, Seymour TL, Glass JM, Fencsik DE, Lauber EJ, Kieras DE, Meyer DE. Virtually perfect time sharing in dual-task performance: Uncorking the central cognitive bottleneck. Psychol Sci. 2001;12(2):101-8.

[55] Ruffieux J, Keller M, Lauber B, Taube W. Changes in standing and walking performance under dual-task conditions across the lifespan. Sports Med. 2015;45(12):1739-58.

[56] Torbeyns T, Bailey S, Bos I, Meeusen R. Active workstations to fight sedentary behaviour. Sports Med. 2014;44(9):1261-73.

[57] Shrestha N, Kukkonen-Harjula KT, Verbeek JH, Ijaz S, Hermans V, Pedisic Z. Workplace interventions for reducing sitting at work. Cochrane Database Syst Rev. 2018;6:CD010912. 